\title{
Giant magneto-impedance in heterogeneous microwires
}

M. Vázquez, ${ }^{\text {a) }}$ J. M. García-Beneytez, and J. M. García

Instituto de Ciencia de Materiales and Instituto de Magnetismo Aplicado, Apdo. 155. 28230 Las Rozas

Madrid, Spain

J. P. Sinnecker

Instituto de Física, Universidade Federal do Rio de Janeiro, C.P. 68528, 21945-970, Rio de Janeiro,

RJ, Brazil

A. P. Zhukov

Donostia International Physics Centre, Paseo Manuel Lardizabal, 4, 20018 San Sebastian and TAMAG S.L. José Abascal, 53, 28003 Madrid, Spain

(Received 6 June 2000; accepted for publication 13 September 2000)

Recent results concerning the giant magneto-impedance effect, GMI, in magnetic microwires having diameter in the range of a few micrometers and heterogeneous character are introduced in this work. Two types of microwires have been studied, namely, (i) magnetic microwires covered by insulating Pyrex glass coating and (ii) copper wires covered by electrodeposited magnetic microtubes. The magnetic anisotropy distribution in each type of microwire is correlated with giant magneto-impedance. While the main source of anisotropy in glass coated microwires is of magnetoelastic origin created by the stresses induced during quenching and by the coating, in the case of the microtubes the origin is the columnar growth during the electrodeposition. The influence on GMI of various treatments that modify the anisotropy is analyzed. Heterogeneous microwires with different thicknesses of insulating coating and of magnetic tube are analyzed. (C) 2000 American Institute of Physics. [S0021-8979(00)04024-X]

\section{INTRODUCTION.}

The giant magneto-impedance, GMI, effect has been recently discovered in magnetically very soft amorphous wires, exhibiting nearly zero magnetostriction constant. ${ }^{1,2}$ Shortly afterwards, observation of this phenomenon has been reported for samples with various geometry (i.e., ribbons and thin films) and extremely soft magnetic character. ${ }^{3,4}$ The present interest lies mainly in the potential applications regarding the development of different kinds of magnetic sensors (as current or position sensors), ${ }^{5,6}$ but also in some interesting fundamental aspects lying behind some technological aspects. ${ }^{7}$ More recently, interest has been also focused on soft magnetic tiny microwires prepared in different ways. ${ }^{8,9}$ In this case, interest is related to their employ in local magnetic field detection technology.

The magnetoimpedance ratio is defined with respect to the maximum applied dc field as follows:

$$
\frac{\Delta Z}{Z}=\frac{Z(H)-Z\left(H_{\max }\right)}{Z\left(H_{\max }\right)} \times 100 .
$$

This change of impedance is a consequence of the modification with that applied field of the skin effect penetration depth, which can be expressed as ${ }^{10}$

$$
\delta=\sqrt{\frac{\rho}{\pi f \mu_{\phi}}},
$$

\footnotetext{
a) Author to whom correspondence should be addressed. Electronic mail: mvazquez@icmm.csic.es
}

where $\mu_{\phi}$ is the circular permeability of the material and $\rho$ is its resistivity. Impedance is typically determined by measuring the voltage drop at the ends of the heterogeneous microwire while keeping constant the amplitude of the ac current of frequency $f$ flowing through it.

The two kinds of magnetic microwires discussed in this work as composite or heterogeneous materials are (i) magnetic microwires covered by a Pyrex-like insulating glass coating and (ii) copper microwires onto which magnetic microtubes were electrodeposited. Each type of microwire exhibits a magnetic anisotropy that can be tailored by modifying the parameters of fabrication and subsequent processings. The objective of this work has been to analyze the influence of the intrinsic magnetic anisotropies on the amplitude of the giant magneto-impedance effect.

\section{GLASS COATED MICROWIRES: INFLUENCE OF STRESSES GENERATED BY THE INSULATING COATING}

The glass coated microwires, currently obtained by a modified Taylor's technique, have the diameter for the amorphous metallic nucleus ranging from about 1 up to $30 \mu \mathrm{m}$ and the coating thickness between 2 and $10 \mu \mathrm{m}$. Their outstanding magnetic properties ${ }^{11,12}$ arise mainly from the magnetoelastic anisotropies induced during the fabrication process by (i) the thermoelastic stresses frozen in during the quenching, (ii) the drawing stresses and, probably most important, (iii) the stresses coming from the difference in expansion coefficient between the insulating coating and the metallic nucleus. ${ }^{13,14}$ The GMI effect has been previously reported in few cases in these microwires exhibiting vanish- 


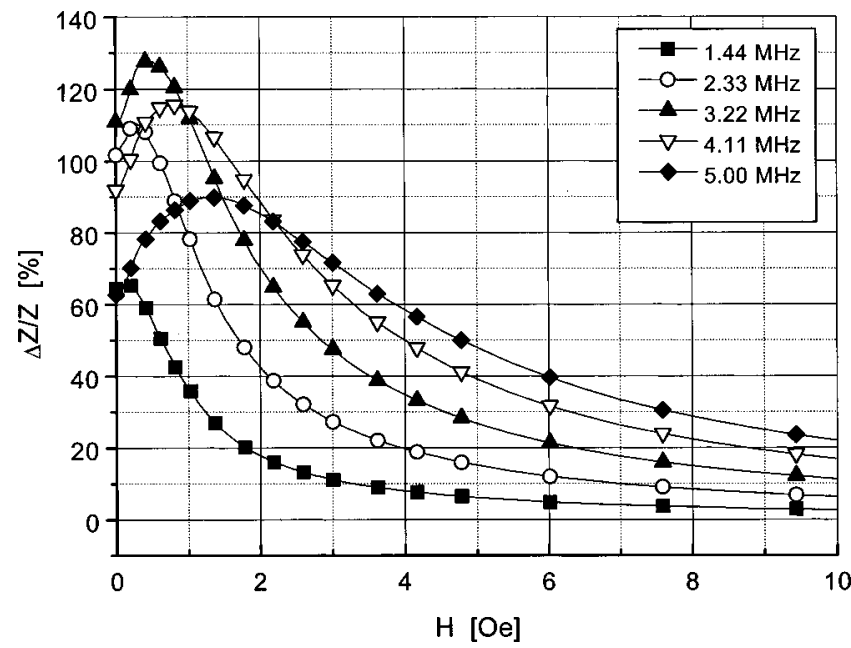

FIG. 1. Magnetoimpedance effect in $\left(\mathrm{Co}_{94} \mathrm{Fe}_{6}\right)_{75} \mathrm{Si}_{10} \mathrm{~B}_{15}$ glass covered microwires for different frequencies. The ac is $1 \mathrm{~mA}_{\mathrm{rms}}$.

ing magnetostriction (within the order of $0.1 \mathrm{ppm}$ ), that is, with the softest magnetic behavior. Results presented here are for nearly nonmagnetostrictive microwires with nominal compositions $\mathrm{Co}_{68.5} \mathrm{Mn}_{6.5} \mathrm{Si}_{10} \mathrm{~B}_{15}$ and $\left(\mathrm{Co}_{94} \mathrm{Fe}_{6}\right)_{75} \mathrm{Si}_{10} \mathrm{~B}_{15}$.

The total impedance of magnetic glass-coated microwires is a complex function depending on the magnetic state of the sample, the electric properties of the material, and the geometry of the sample under study. All this can be studied through the dependence of the impedance as a function of three external parameters: the external dc magnetic field, the current flowing through the sample, and its frequency. Figure 1 shows the dependence of the impedance with the external magnetic field for different frequencies within the range 1-5 $\mathrm{MHz}$. In all the measurements, the intensity of the ac has been kept constant and equal to $1 \mathrm{~mA}$ (rms value).

The results in Fig. 1 show the general features of MI curves. Note the observed amplitude of the GMI, as large as $130 \%$. Typically, the impedance reaches a maximum in the low-field region at $H=H_{k}$, followed by a decrease up to saturation at large applied fields. This maximum is ascribed to the existence of a circular magnetic anisotropy of magnetoelastic nature. As the applied axial field reaches the circular anisotropy field, $H_{k}$, a maximum for the transverse permeability and, consequently, for the MI effect as well is obtained. As observed, the maximum MI shifts towards higher fields as the working frequency increases. This has been associated to the radial distribution of the circular anisotropy. ${ }^{15}$ For increasing frequency the skin effect penetration depth decreases so that the region of the material involved in the MI effect itself approaches its surface where the anisotropy field is higher due to stronger internal stresses.

The origin of this magnetoelastic anisotropy is found in the internal stresses produced during quenching procedure. However, if the samples are submitted to thermal treatments in a suitable range of temperatures, it is possible to observe the relaxation of those internal stresses. This relaxation leads to a decrease in the strength of the transverse anisotropy as well as to its associated anisotropy field, $H_{k}$, as can be observed in the MI curves in Fig. 2.

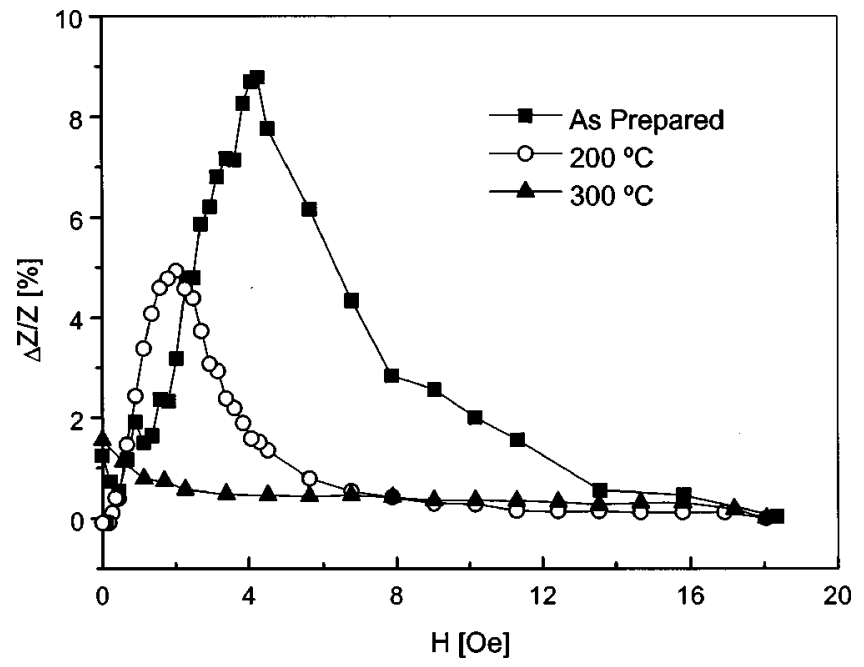

FIG. 2. MI curves measured at a frequency of $200 \mathrm{kHz}$ for as-prepared $\mathrm{CoMnSiB}$ microwires and after being submitted to treatments at indicated temperatures.

The circular anisotropy field for the as-prepared sample is around 5 Oe. If we now anneal the sample at $200^{\circ} \mathrm{C}$, the peak moves towards smaller values of the field $(2 \mathrm{Oe})$, indicating the partial relaxation of stresses. Annealing at higher temperature leads to a further decrease of the maximum, which eventually disappears when the annealing is performed at $350^{\circ} \mathrm{C}$.

The influence of the stresses generated by the glass coating is further analyzed by considering microwires with different relative thicknesses of the metallic nucleus and the external glass coating. This is achieved through the control of the fabrication parameters (winding velocity, temperature of the melt, drawing stress, etc.). Figure 3 shows typical MI curves for two microwires with the same diameter of the nucleus but different thicknesses of the coating.

It is expected that a thicker coating would lead to larger stresses and to higher values of the induced circular anisotropy. In fact, in the sample with smaller glass coating thickness, the peak of the MI is located at 0.4 Oe. In the sample with thicker glass coating, the anisotropy field rises to 2.6 Oe. Furthermore, the coating does not only vary the anisotropy distribution, but it also reduces the value of the magnetic permeability (reduces the "softness" of the magnetic sample). Consequently, a reduction in the maximum variation of the impedance with the field is observed.

When the frequency of the ac excitation is larger than about $10 \mathrm{MHz}$, the conventional measuring methods start to be inadequate and experimental techniques specially designed for measurements in the microwave regime must be recalled. Usually, these alternative techniques are based on the determination of the scattering parameters $S$ of a coaxial line (by network analyzer) which has been modified, introducing the magnetic samples under study as active elements of the line. ${ }^{16-18}$ An example is given in Fig. 4(a) for frequencies around $1 \mathrm{GHz}$. As observed the MI curve at $0.92 \mathrm{GHz}$ shows the typical two-peak pattern associated to a circular anisotropy. However, at higher frequencies, the shape of the curve modified and only the initial increase of impedance 

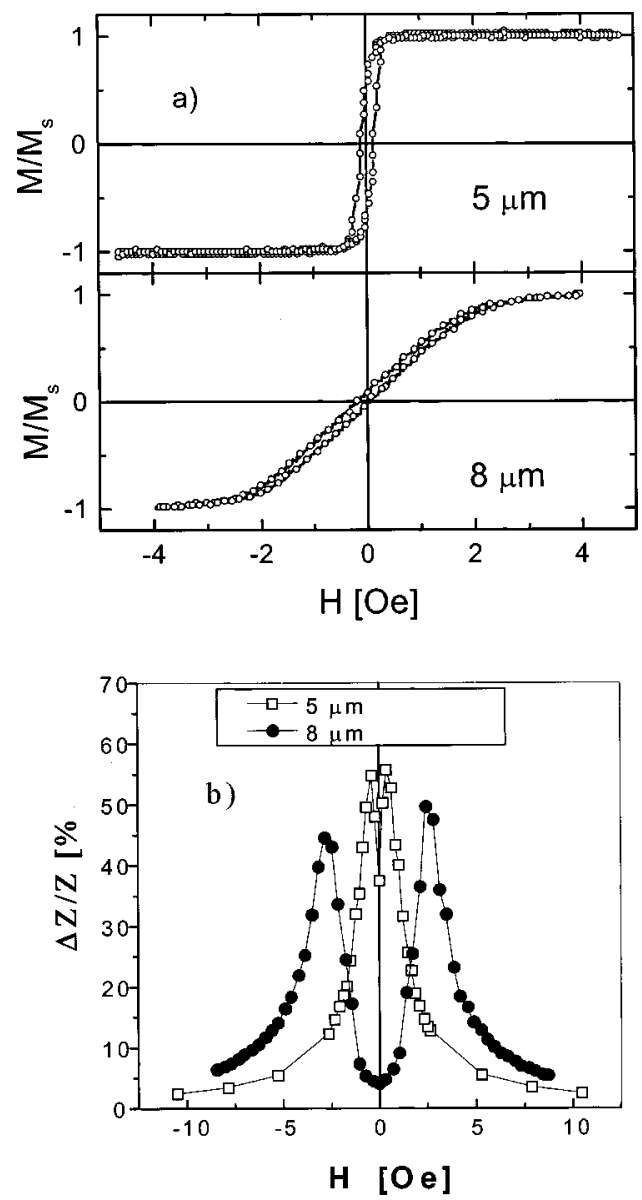

FIG. 3. (a) Hysteresis and (b) MI curves for CoSiMnB glass covered microwires, with the same diameter of the metallic nucleus $(15 \mu)$ but different thickness of the glass coating (5 and $8 \mu \mathrm{m}$ ). The frequency of the measurement is $2 \mathrm{MHz}$.

with applied field is observed for this range of applied field.

It is also interesting to study the low-field behavior of the magnetoimpedance effect in the microwave region. Measuring techniques involve the determination of microwave losses through cavity perturbations At these frequencies, the ferromagnetic resonance phenomena take place for fields of the order of kOe, so variations of the impedance appearing at fields of units of Oe or less cannot be ascribed to conventional FMR. In Fig. 4(b), the relative change in loss is plotted as a function of the external field, applied parallel to the axis of the sample, and normal to the exciting rf magnetic field. ${ }^{19}$ Even at these high frequencies, the peaks associated with the transverse anisotropy induced by the presence of the external coating are present and can be detected.

\section{MICROTUBES ELECTRODEPOSITED ON CU WIRES: COLUMNAR GROWTH MAGNETIC ANISOTROPY}

Electrodeposition is a well-known technique used for fabrication of amorphous alloys in the shape of ribbons and tubes. After discovering the GMI in amorphous wires, this technique has recently been employed to deposit magnetic tubes on $\mathrm{Cu}$ wires which act as electrodes in the deposition. These magnetic coatings show a typical circular magnetic
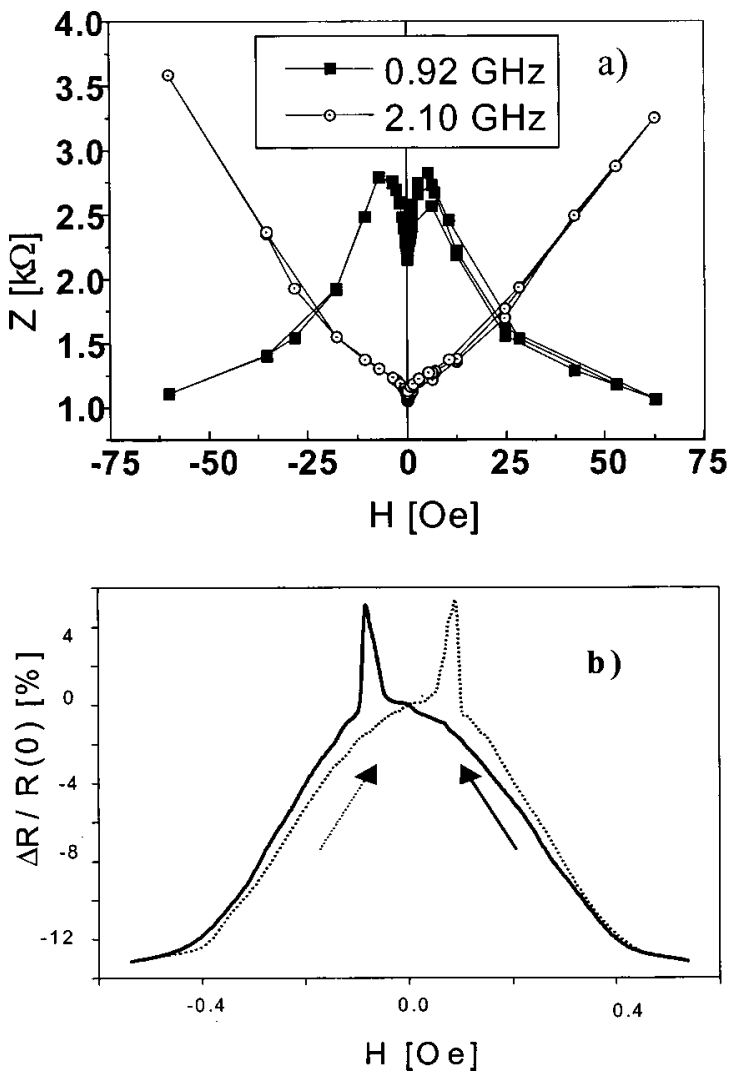

FIG. 4. High frequency magnetoimpedance. (a) Field dependence of impedance in $\mathrm{CoMnSiB}$ microwires at 0.92 and $2.10 \mathrm{GHz}$. (b) Low-field behavior of magnetic losses at $9.8 \mathrm{GHz}$.

anisotropy. ${ }^{9,20}$ Now, in this work we present a new type of sample: $\mathrm{CoP}$ microtubes electrolytically grown over $\mathrm{Cu}$ wires. The main novelty is that these samples exhibit the GMI effect even though they have radial anisotropy. ${ }^{21}$

Layers of amorphous $\mathrm{Co}_{90} \mathrm{P}_{10}$ with a thickness ranging from 2 to $20 \mu \mathrm{m}$ have been electrodeposited onto $\mathrm{Cu}$ wires (200 $\mu \mathrm{m}$ diameter). The thickness of the CoP layer is controlled using different deposition times, and its amorphous character was verified by means of x-ray diffraction. As it has been previously reported, ${ }^{22-24}$ the electrodeposition of Co-P amorphous alloys gives rise to anisotropic materials, the anisotropy being either planar or perpendicular depending on the layer thickness. For layer thickness below $0.4 \mu \mathrm{m}$, the anisotropy is planar. On the other hand, when the thickness is of several $\mu \mathrm{m}$, the anisotropy is mainly perpendicular to the surface owing to the columnar growth characteristic for electrodeposited films. Taking into account the cylindrical geometry of our substrates, the out-of-plane anisotropy will lead to a uniaxial magnetic anisotropy with the easy axis in the radial direction.

Figures 5(a) and 5(b) show, respectively, the longitudinal hysteresis loop for a $20 \mu \mathrm{m}$ thick microtube and its probability distribution function $P(H)$ of anisotropy fields which has been calculated using the second derivative method. ${ }^{25}$ According to this figure, the easy magnetization axis has certainly a main perpendicular direction with regards to the longitudinal direction, and an anisotropy field of around 60 Oe can be deduced. MFM images prove that the samples 

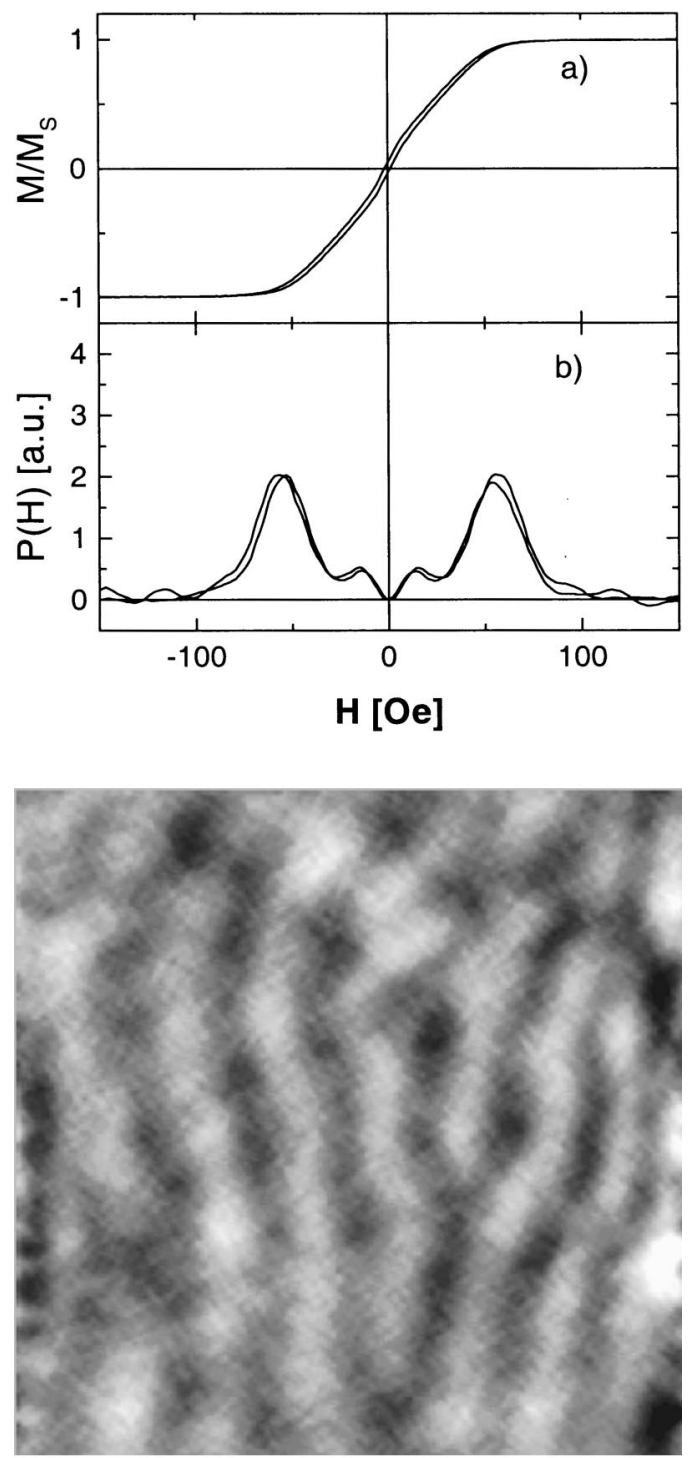

c)

FIG. 5. (a) Longitudinal hysteresis loop and (b) anisotropy field probability distribution function for a sample with $20 \mu \mathrm{m}$ thickness of CoP layer; (c) MFM image of a $4 \mu \mathrm{m}$ thick sample. Image size: $38 \times 38 \mu \mathrm{m}^{2}$. The axis of the microtube was oriented along the horizontal direction of the image.

have a radial anisotropy, as shown in Fig. 5(c). This domain pattern is typical for materials exhibiting an out-of-the-plane anisotropy, with the dark and bright areas ascribed to domains with up and down magnetization

Figure 6(a) shows the magnetoimpedance ratio for different samples measured using an ac of $10 \mathrm{~mA}_{\mathrm{rms}}$ at $1 \mathrm{MHz}$. The maximum value of the $\Delta Z / Z$ ratio increases with layer thickness from approximately $2.9 \%$ up to $16.6 \%$ for the 2 and $20 \mu \mathrm{m}$ layers, respectively. This behavior can be explained considering that the magnetoimpedance effect is related to changes in the classical current penetration depth. One should consider that in the sample with the highest layer thickness, the total cross section of the wire is the biggest. So, as the magnetoimpedance is related to the change of the cross section actually seen by the current, in the sample with the biggest cross section we observe the highest change in the $\Delta Z / Z$ ratio.
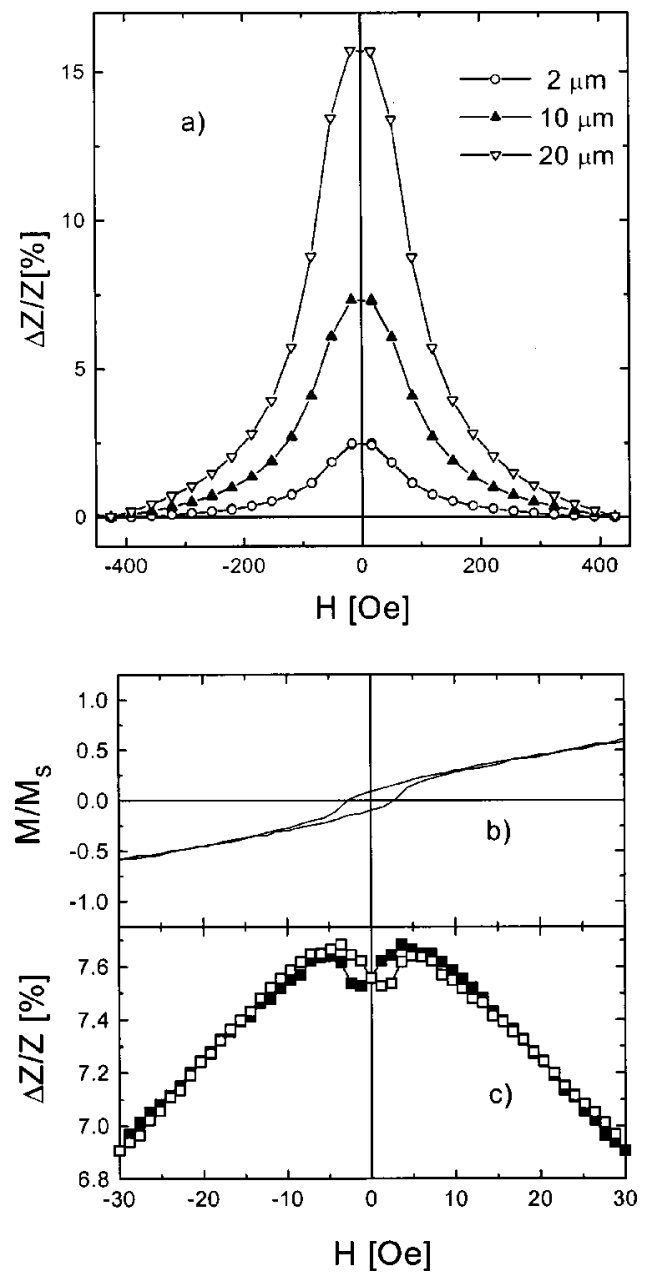

FIG. 6. (a) GMI ratio for a $10 \mathrm{~mA} / 1 \mathrm{MHz}$ ac probe in samples with different CoP layer thicknesses: 2, 10 and $20 \mu \mathrm{m}$. (b) Hysteresis loop and (c) GMI ratio evolution with a $10 \mathrm{~mA} / 1 \mathrm{MHz}$ ac for a sample with $10 \mu \mathrm{m}$ thickness in the low-field region.

By analyzing carefully the low field region in both the magnetization and the magnetoimpedance curves, we can identify another peculiarity of these samples. In Figs. 6(b) and 6(c) the $\Delta Z / Z$ ratio and the hysteresis loop of a $10 \mu \mathrm{m}$ thick microtube are displayed for a field region up to 30 Oe . A hysteresis is observed in this low field region, with coercivity of about $4 \mathrm{Oe}$. The $\Delta Z / Z$ curve exhibits peaks at an applied field close to the coercivity, and such peaks should be related to the still remaining irreversible magnetization process by domain wall displacements. ${ }^{26}$

It is worth noticing that the maximum of the GMI effect does not fit to the transverse anisotropy field as do conventional wires with circular transverse anisotropy. Actually, maximum GMI is achieved for a magnetic field of the order of the irreversibility field (roughly coercivity, tenths of kA/ $\mathrm{m})$. This should be ascribed to the radial easy axis, and it is very promising since the two-peak behavior (undesirable for applications) practically disappears in the hole GMI curves where the applied fields reach several tens of $\mathrm{kA} / \mathrm{m}$.

\section{CONCLUSIONS}

The giant magnetoimpedance effect has been studied in two families of heterogeneous amorphous magnetic microw- 
ires with composition rich in Co and small additions of other metals such as Fe or Mn that drastically reduce the magnetostriction constant. Magnetoimpedance has been proved to be strongly dependent on the magnetic anisotropies that are introduced by the particular fabrication method. GMI is quite a promising effect for its application in sensing devices where high (i.e., high-current sensors) or local (recording media) magnetic field detection is required.

\section{ACKNOWLEDGMENTS}

The authors wish to thank Dr. A. Asenjo for MFM imaging. This work has been performed under Spanish projects CAM/07N/0033/1998 and CICYT MAT98-0965-C04.

${ }^{1}$ L. V. Panina and K. Mohri, Appl. Phys. Lett. 65, 1189 (1994).

${ }^{2}$ R. S. Beach and A. E. Berkowitz, Appl. Phys. Lett. 64, 3652 (1994).

${ }^{3}$ M. Tejedor, B. Hernando, M. L. Sánchez, and A. García-Arribas, J. Magn. Magn. Mater. 157/158, 141 (1996).

${ }^{4}$ R. L. Sommer and C. L. Chien, Appl. Phys. Lett. 67, 3346 (1995).

${ }^{5}$ M. Vázquez, M. Knobel, M. L. Sánchez, R. Valenzuela, and A. P. Zhukov, Sens. Actuators 59, 20 (1997).

${ }^{6}$ K. Mohri, T. Uchiyama, and L. V. Panina, Sens. Actuators 59, 1 (1997).

${ }^{7}$ A. Yelon, D. Ménard, M. Britel, and P. Ciureanu, Appl. Phys. Lett. 69, 3084 (1996).

${ }^{8}$ R. S. Beach, N. Smith, C. L. Platt, F. Jeffers, and A. E. Berkowitz, Appl. Phys. Lett. 68, 2753 (1996).

${ }^{9}$ M. Vázquez, A. P. Zhukov, P. Aragoneses, J. Arcas, J. M. GarcíaBeneytez, P. Marín, and A. Hernando, IEEE Trans. Magn. 34, 724 (1998).

${ }^{10}$ L. D. Landau, E. M. Lifshitz, and L. P. Pitaevskii, Electrodynamics of Continuous Media - Course of Theoretical Physics: Vol. 8 (Butterworth-
Heinemann Ltd.-Linacre House, Jordan Hill, Oxford, England, 1995), p. 208.

${ }^{11}$ M. Vázquez and A. P. Zhukov, J. Magn. Magn. Mater. 160, 223 (1996).

${ }^{12} \mathrm{H}$. Chiriac and T. A. Ovari, in Progress in Materials Science (Elsevier, London, 1997), pp. 40, 333.

${ }^{13}$ J. Velázquez, M. Vázquez, and A. P. Zhukov, J. Mater. Res. 11, 2499 (1996).

${ }^{14}$ H. Chiriac, T. A. Ovari, and G. Pop, Phys. Rev. B 52, 10 1041995).

${ }^{15}$ J. M. García-Beneytez, F. Vinai, L. Brunetti, H. García-Miquel, and M. Vázquez, Sens. Actuators 81, 78 (2000).

${ }^{16}$ D. Ménard, M. Britel, P. Ciureanu, A. Yelon, V. P. Paramonov, A. S. Antonov, P. Rudkowski, and J. O. Ström-Olsen, J. Appl. Phys. 81, 4032 (1997).

${ }^{17}$ O. Acher, P. M. Jacquart, J. M. Fontaine, P. Baclet, and G. Perrin, IEEE Trans. Magn. 30, 4533 (1994).

${ }^{18}$ H. García-Miquel, M. J. Esbrí, J. M. Andrés, J. M. García, J. M. GarcíaBeneytez and M. Vázquez, IEEE Trans. Magn. (in press).

${ }^{19}$ S. E. Lofland, S. M. Baghat, M. Dominguez, J. M. García-Beneytez, F. Guerrero, and M. Vázquez, J. Appl. Phys. 85, 4442 (1998).

${ }^{20}$ N. Usov, A. Antonov, and A. Granovsky, J. Magn. Magn. Mater. 171, 64 (1997)

${ }^{21}$ J. P. Sinnecker, J. M. García, A. Asenjo, M. Vázquez, and A. GarciaArribas, J. Mater. Res. 15, 751 (2000).

${ }^{22}$ G. S. Cargil III, R. J. Gambino, and J. J. Cuomo, IEEE Trans. Magn. 10, 803 (1974).

${ }^{23}$ G. Dietz, H. Bestgen, and J. Hungenberg, J. Magn. Magn. Mater. 9, 208 (1978).

${ }^{24}$ J. M. Riveiro and M. C. Sánchez-Trujillo, IEEE Trans. Magn. MAG16(6), 1426 (1980).

${ }^{25}$ J. M. Barandiarán, M. Vázquez, A. Hernando, J. González, and G. Rivero, IEEE Trans. Magn. 25, 3330 (1989).

${ }^{26}$ D. X. Chen, J. L. Muñoz, A. Hernando, and M. Vázquez, Phys. Rev. B 57, $106991998)$ 